\title{
EMG Biofeedback In Patients With Motor Disorders: An Aid For Co-Ordinating Activity In Antagonistic Muscle Groups
}

\author{
ALAN E. DAVIS AND ROBERT G. LEE
}

SUMMARY: A computer program was developed to analyse the relative amount of EMG activity in an agonist-antagonist pair of muscles while subjects performed voluntary flexion-extension movements at the wrist to track a visual target. The data were presented to the subjects in the form of a vector display, the angle and length of which was determined from calculation of EMG power in the two muscles.

RESUME: Nous avons développé un programme d'informatique afin d'analyser la quantité relative d'activité EMG dans une paire de muscles agonistes/antagonistes alors que les sujets effectuaient des mouvements volontaires de flexion-extension du poignet de façon à suivre une cible visuelle. Les données furent présentées aux sujets sous la forme d'un tableau vectoriel dont l'angle et la longueur étaient déterminés à partir du calcul de la puissance EMG dans les deux muscles.
This new approach to EMG biofeedback was evaluated in two hemiplegic patients and three patients with cerebellar incoordination. Over a training period of several weeks, all the subjects were able to modify' the pattern of EMG activity in the muscles to reduce the amount of inappropriate coactivation of flexors and extensors and to produce more sustained and regular acrivation of individual muscle groups.

Cette nouvelle approche de rétrocontrôle EMG fut évaluée chez deux patients hémiplégiques et trois patients avec incoordination cérébelleuse. Après une période d'entraînement de plusieurs semaines, tous les sujets purent modifier le pattern d'activité EMG dans les muscles éludiés afin d'ainsi réduire la quantité de coactivation inappropriée des fléchisseurs et extenseurs et afin de produire une activation des groupes musculaires individuels soutenue et régulière.
From The Neuroscience Research Group. Faculty of Medicine, University of Calgary.

Reprint requests to Dr. Robert G. Lee, Faculty of Medicine, University of Calgary. Calgary. Alberta, T2N IN4. Canada.

\section{INTRODUCTION}

Biofeedback of physiological parameters of various types has acquired some popularity in the management of certain neurologic disorders (see Basmajian, 1979). Several groups of investigators have employed feedback of electromyographic (EMG) activity as an adjunct to conventional physiotherapy in the rehabilitation of motor disorders such as hemiplegia (Brudny et al, 1974; Basmajian et al, 1975; Lee et al, 1976; Basmajian et al, 1977), torticollis and related dystonic syndromes (Brudny et al, 1976; Korein and Brudny, 1976; Bird and Cataldo, 1978) and tardive dyskinesia (Albanese and Gaardner, 1977). The usual approach has been to provide the patient with a visual or auditory signal representing EMG activity recorded from a single muscle. The patient's task is to either minimize the signal level to reduce spasticity or involuntary movements or to increase the signal to reinforce attempts to activate paretic muscles. A limitation of this "one-dimensional" approach is that some motor disorders are characterized by defective interaction between opposing groups of muscles (Terzuolo et al, 1973). Under normal conditions, voluntary activation of a muscle group is associated with reciprocal inhibition of its antagonists (Hultborn et al, 1973) but in hemiplegic patients and patients with cerebellar incoordination we have frequently observed examples of abnormal coactivation of agonist and antagonist muscles. To improve motor performance these patients are faced with two tasks. At the same time as they are activating weak or poorly coordinated muscles they must learn to suppress inappropriate activity from antagonistic muscles. 
The present study was undertaken to determine whether biofeedback of a preprocessed signal providing information concerning the relative amount of EMG activity in two opposing muscle groups could assist patients with motor deficits in carrying out a simple motor task. A small laboratory computer system (PDP 11-40) was used to carry out on-line analysis of EMG activity from the wrist flexors and extensors and to generate a visual display on an oscilloscope. Detailed studies were carried out on five patients - two with spastic hemiplegia following cerebral infarcts and three with cerebellar incoordination. With feed back training all five patients were able, over a period of time, to modify and improve the pattern of EMG activity in the muscles studied.

\section{MATERIALS AND METHODS}

\section{Clinical Material}

a) Patients with spastic hemiplegia Case 1: A 39-year-old right handed woman presented with a subarachnoid hemorrhage due to rupture of a right internal carotid artery aneurysm. Surgical clipping of the aneurysm was complicated by postoperative occlusion of the right internal carotid artery resulting in a cerebral infarct with severe left hemiparesis. Some improvement occurred over the next several months but at the time biofeedback studies were commenced 14 months following the onset of her illness she still had moderate weakness in most muscle groups in the left arm, spasticity in the wrist and finger flexors, and inability to control movement of individual fingers. Attempts to perform rapid flexionextension movements of the wrist were slow and irregular. Position sense was diminished in the fingers of the left hand.

Case 2: A 51-year-old right handed man developed a right fronto-parietal cerebral infarct due to thrombotic occlusion of the right internal carotid artery. Initially he had severe left hemiplegia with complete paralysis of the arm. With intensive physiotherapy he regained the ability to walk, but there was only limited recovery in the arm. Biofeedback training was commenced ten months following the stroke. At that time the left arm was paralyzed except for slight movement at the shoulder and a minimal degree of wrist flexion. There was marked spasticity in the wrist flexors. Sensation was impaired for all modalities in the left forearm and hand.

b) Patients with cerebellar incoordination

Case 3: A 34-year-old left handed woman presented with a 14 year history of relapsing and remitting neurologic symptoms characteristic of multiple sclerosis. At the time of our study there was clinical evidence of lesions at multiple sites including the optic nerves, brain stem, and spinal cord. Her major deficit was a spastic paraplegia, but for several years she had shown evidence of a cerebellar deficit in the left arm with incoordination on the finger-nose test, mild intention tremor, and moderate slowing of rapid alternating movements. Muscle tone in the left arm was slightly reduced. Sensation in the upper extremities was normal. Case 4: A 64-year-old right handed man had a 15 year history of progressive spinocerebellar degeneration of undetermined type. He was confined to a wheelchair because of severe trunkal ataxia. Examination of the upper extremities revealed severe intention tremor and incoordination. Rapid alternating movements were extremely slow and clumsy. Muscle strength in the arms was normal except for slight weakness of the intrinsic hand muscles due to an associated peripheral neuropathy. Sensory examination was normal.

Case 5: A 29-year-old right handed woman had a 13 year history of Friedreich's ataxia. She exhibited marked dysmetria on performing the finger-nose test with either hand. Rapid alternating movements were slow and clumsy. Muscle tone was reduced and tendon reflexes were absent in all four extremities. Position sense was slightly diminished in the fingers.

2. EMG Recording and Generation of Display

Recordings were carried out with the subjects seated in a chair facing the video terminal (GT 40) of a PDP $11-40$ computer. The arm rested on a manipulandum which kept the elbow flexed to $90^{\circ}$ and restricted movement to the wrist joint. The subject's hand was taped firmly to a handle which permitted voluntary flexion and extension of the wrist through a range of approximately $90^{\circ}$. A potentiometer monitored changes in position of the handle.
EMG activity was recorded with pairs of surface electrodes applied $3.5 \mathrm{~cm}$ apart over the flexor carpi radialis and extensor carpi ulnaris muscles. After appropriate amplification, the EMG signals were sampled on line at a rate of $400 \mathrm{~Hz}$. The "power" or variance of the signal about a mean value was computed for each channel over 100 msec. intervals. The ratio of flexor EMG power to extensor EMG power was used to generate a vector display on the video terminal. Details of the calculations resulting in the vector are provided in the appendix.

Figure 1 illustrates the display presented to the subject while carrying out flexion and extension movements of the left wrist. When the subject moves the handle to the right (figure 1-A) flexor EMG power exceeds extensor EMG power and the vector is tilted to the right. The opposite situation is shown in figure 1-C where the subject is extending the wrist to move the handle to the left. When the amount of EMG activity is equal in flexors and extensors, as in figure 1-B, the vector assumes a vertical direction.

It is important to note than the angle of the vector is not directly related to handle position, but rather reflects the relative degree of activation of flexor and extensor muscle groups. If the subject activates the flexors and totally inhibits the extensors the vector will assume a horizontal position directed to the right of the screen.

In addition to controlling the direction of the vector the subject is able to control its length by varying the total amount of EMG activity. Thus, a strong contraction of either or both muscle groups produces a long vector while complete relaxation reduces the vector to a very short line or to a point display.

\section{Biofeedback Training Sequence}

Training was initiated by having the computer generate a $4 \mathrm{~cm} \times 4 \mathrm{~cm}$ square target on the video display (figure 1, D-F). The subjects were instructed to experiment with movements of the handle and contraction of the forearm muscles until they were able to locate and hold the end of the vector within the target area. Once this was accomplished the computer program shifted the target $1 \mathrm{~mm}$. to the right and the sequence was repeated. The target continued to move across the screen in $1 \mathrm{~mm}$. steps until it reached the edge of the display, at which time it began to move back in the opposite direction. Each step in the sequence occurred only after the subject had successfully completed the previous step. The speed at which the target moved was determined by the accuracy and skill with which the subject was able to generate the appropriate level of EMG 
activity in wrist flexors and extensors to maintain the vector in the target area. Simple modifications in the program permitted the investigator to vary the complexity of the motor task to match the needs of individual patients. Although the velocity of target movement was not identical for all the patients, it was kept constant for each individual over the entire training period.
Following initial familiarization with the equipment and the video display, patients returned to the laboratory two or three times a week for biofeedback training sessions lasting approximately one hour. During these sessions the subjects carried out repeated 5 minute runs of tracking the visual target, each of which was followed by a 5 minute session during which they were instructed to carry out slow wrist
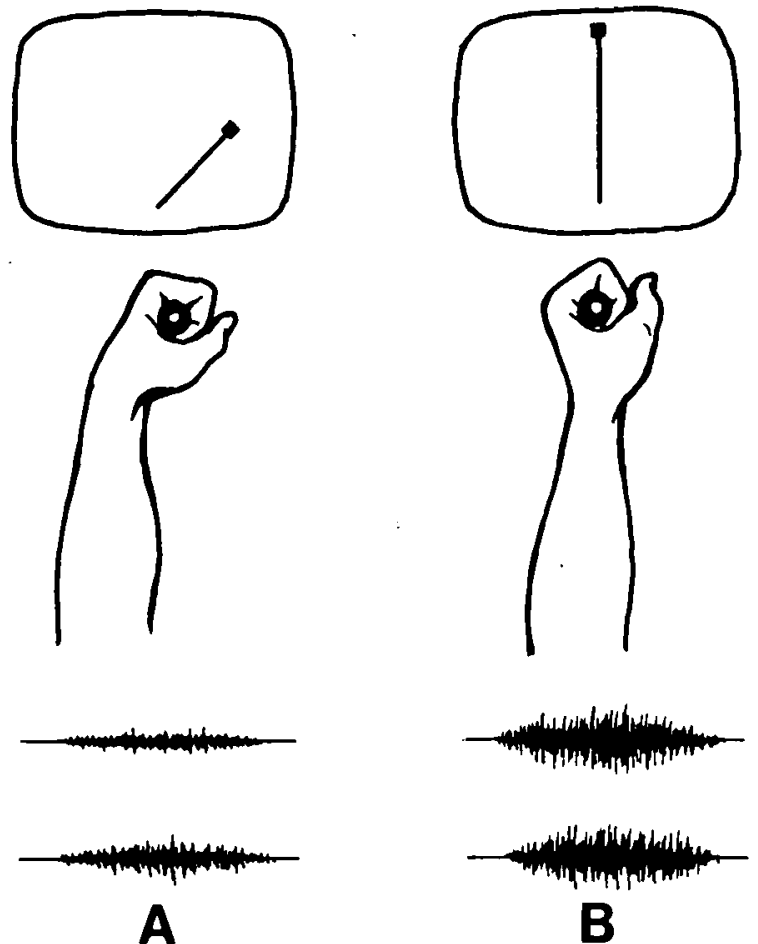

A
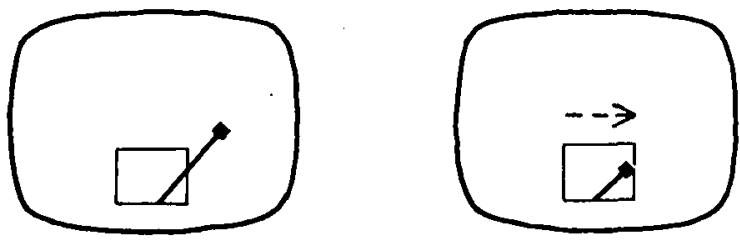

E

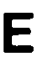

Figure 1 - Examples of vector display provided to the patients during biofeedback training of wrist movements and relationship to EMG activity produced by a subject moving his left wrist. Vector tilts to the right when flex or EMG power exceeds extensor EMG power (A), to the left when extensor EMG power exceeds flexor EMG power (C). Vector assumes a vertical position when flexor EMG power equals extensor EMG power. The length of the vector is determined by the absolute sum of EMG activity in the two channels.

The computer controlled target which moves back and forth across the display is illustrated in D-F.

flexion-extension movements at a similar rate but without visual feedback. Recordings of EMG and handle position during these "no-feedback" runs were compared with the data obtained during visual feedback. Each individual run was followed by a three minute rest period. In addition, at the conclusion of each session the subjects were instructed to carry out a short sequence of flexion-extension movements as rapidly as possible without visual feedback.

\section{Data Analysis}

In addition to controlling the EMG vector display and target movement, the computer program recorded the time spent in flexion, extension and coactivation as the patient tracked the target back and forth across the screen. Flexion was defined as the time during which flexor EMG power was greater than 1.5 times extensor EMG power and extension was when extensor EMG power exceeded flexor EMG power by a similar amount. Coactivation was defined as the remainder, that is the time when flexor and extensor EMG power differed by less than $50 \%$.

\section{RESULTS}

\section{Hemiplegic Patients}

Figure 2 shows examples of EMG activity and associated handle movement for the two hemiplegic patients during the first recording session and at the end of a two month training period. The initial attempts by Case 1 to produce smooth flexion-extension movements using biofeedback are characterized by irregular bursts of EMG activity from the flexors without any well defined activity from the extensors (figure 2-A). Similar recording at the end of the training period, shown on the right, reveals that the patient has developed the ability to produce reciprocal activation of flexors and extensors to generate relatively smooth movements of the handle.

Figure 2-B illustrates attempts by case 1 to produce rapid flexionextension movements without visual feedback. During the first session these movements were slow and irregular and coactivation of extensors and flexors occurred commonly. Following the training period the frequency and rhythmicity of these movements was increased. 

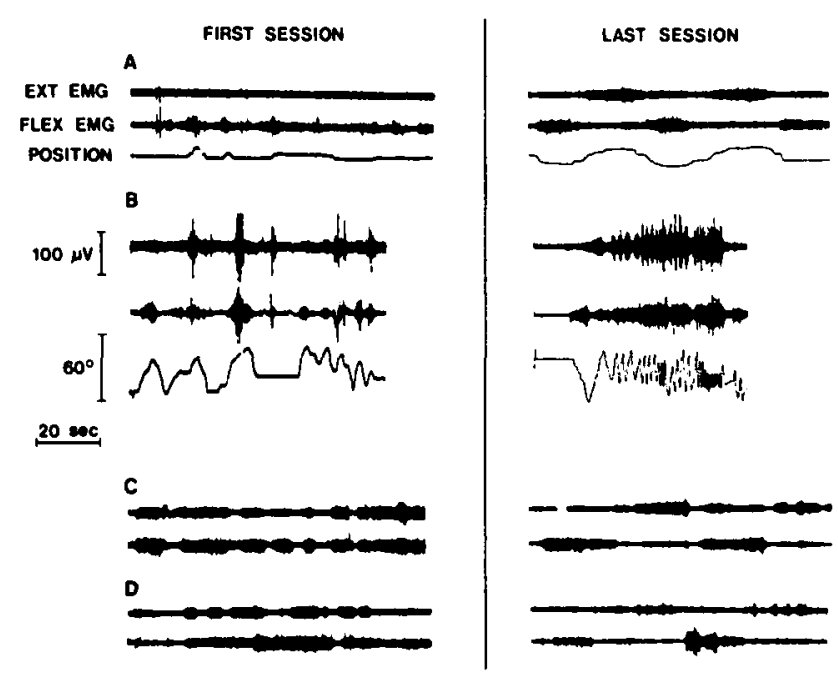

Figure 2 - Recordings of surface EMG from wrist flexors and extensors and handle position in two hemiplegic patients performing a wrist flexion-extension task. Recordings on the left were obtained at the first biofeedback session, those on the right after two months of feedback training. Tracings A and B are from case 1 . A shows subject's attempt to perform smooth tracking of the visual target. B represents attempts to carry out rapid wrist flexion-extension without visual feedback. Tracings $C$ and $D$ are from case 2 with visual feedback (C) and without feedback (D). Handle position is not shown for case 2 as he did not have sufficient strength in the forearm muscles to generate recordable movement of the handle.

Upward deflection in the handle position trace represents wrist flexion in this and subsequent illustrations.
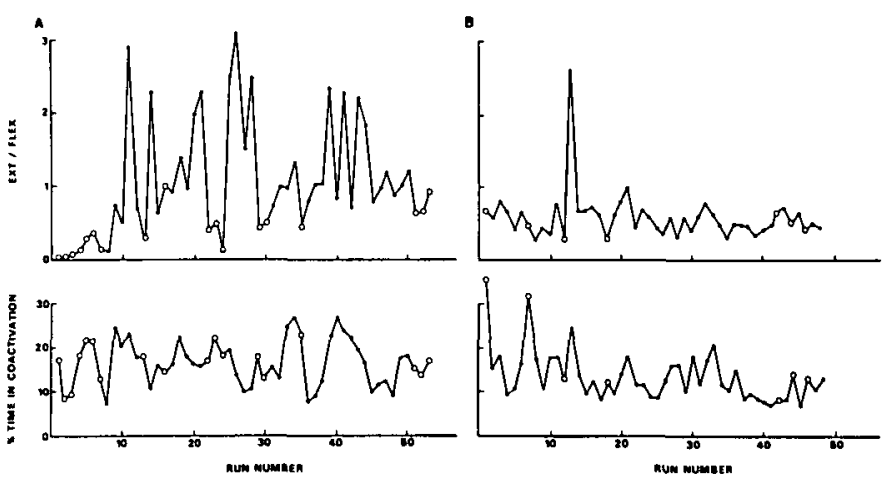

Figure 3 - Relationship between activation of wrist extensors and wrist flexors and percentage of time during which these muscles were coactivated for two hemiplegic patients, case 1 (A) and case 2 (B). Each point on the graphs represents results for one five minute run. Closed circles represent runs with visual feedback, open circles runs with no feedback. The total time period represented on the abscissa is approximately three months. The last five points on each graph were obtained at the one month followup session after completion of the training sequence. Value for the ordinates in the upper graphs were obtained by calculating for each run the following ratio:

Time extensor EMG power is 1.5 times > flexor EMG power Time flexor EMG power is 1.5 times $>$ extensor EMG power (see methods)
Case 2 had marked paresis of the hemiplegic arm, and although he was able to activate EMG discharges these were not sufficient to produce any appreciable movement of the handle. Nevertheless EMG recordings with feedback (figure 2-C) and without feedback (figure 2-D) show some improvement in the ability to suppress coactivation and to selectively control either flexor or extensor muscles in the forearm following several weeks of biofeedback training. The training in this patient, however, did not result in significant functional improvement in control of movement.

To quantitate the effect of biofeedback on the ability of the patients to control flexor and extensor muscles independently a ratio was calculated for each five minute run to indicate the relative amount of time during which either flexors or extensors were predominantly active. The percentage of total time during which there was coactivation of flexors and extensors was also determined. The results for the two hemiplegic patients over the two month training period are shown in figure 3.

Case 1 initially showed a marked predominance of flexor activation resulting in very small extensor/flexor ratios (figure 3-A, top). With biofeedback she was able to produce more activation of the extensors, and over the course of the training period the extensor-flexor ratios gradually approached the optimal value of one which represents balanced activity in these opposing muscle groups. This change was present even in runs without feedback and was maintained during the follow-up session one month later.

The bottom graph in figure 3-A shows that there was coactivation of flexors and extensors between 10 and $25 \%$ of the total time. This did not change during the training period although there was some tendency for coactivation to be less during runs with feedback.
In contrast, case 2 showed a gradual reduction in the proportion of time during which flexors and extensors were coactivated, and this effect was still present at the one month followup (figure 3-B, bottom). The extensor/ flexor ratios for this patient remained relatively constant throughout the recording sessions.

\section{Patients With Cerebellar Incoordination}

Figure 4 shows recordings of EMG and handle position for case 3 , a patient with multiple sclerosis causing moderately severe dysmetria of the left arm. Prior to training (figure 4-A) bursts of EMG activity were irregular and prolonged and the resulting handle movements were slow and jerky. After eight sessions of feedback training over a five week period the patient was able to generate more discrete bursts of EMG activity and handle movements were faster and of greater amplitude. The recordings on the right of figure 4 show the patient's 
attempts to carry out rapid wrist flexion-extension movements without feedback. The maximum frequency of these movements was considerably increased after five weeks of training.

Results for case 4 , a patient with spinocerebellar degeneration and severe intention tremor, are shown in figure 5. The initial recording (figure 5A) shows inappropriate coactivation of extensors and flexors with slow irregular movements of the handle. After four feedback sessions, each consisting of three five minute runs, there was less coactivation and handle movements were more regular (Figure 5-B). In addition the patient was able to maintain this more normal pattern of movement during voluntary flexion and extension of the wrist without feedback (figure 5-C). The effect of biofeedback on this patient's motor activity is illustrated further in figure 6-A which illustrates graphically the relative amounts of flexor and extensor activation and coactivation of both muscle groups during sequential runs with and without feedback. The amount of coactivation is clearly reduced during runs with feedback.

The data for case 5 , a patient with Friedreich's ataxia, are illustrated in a similar format in figure 6-B. With this patient an attempt was made to determine whether the improved performance with feedback was directly related to the information provided by the feedback or whether it might be due to other associated factors such as increased attention or motivation. To investigate this possibility a distorted form of feedback was introduced on some runs. The length and angle of the vector display were randomly scaled every $100 \mathrm{msec}$. using a range of plus or minus $25 \%$ of the actual values determined from the calculation of EMG power. This patient was given the same instructions as the other patients and was not informed when the feedback was being distorted.

Figure 6-B shows that without feedback this patient had difficulty producing any activation of flexor muscles. With distorted feedback there was some increase in the percentage of total time during which the flexors were activated, but this was less than what occurred when she was provided with true feedback, which resulted in the flexors being activated at least $50 \%$ of the time. This suggests that she was able to use the specific information provided by the true feedback to assist her in activating the flexor muscles.

\section{DISCUSSION}

These studies show that with visual feedback of information concerning relative activation of antagonistic muscle groups patients with certain motor deficits are able to modify the patterns of $E M G$ activity in these muscles. Patients with either hemiplegia or cerebellar incoordination were able to utilize the feedback to reduce the amount of inappropriate coactivation of flexors and extensors and to produce more sustained and regular activation of individual muscle groups during a simple wrist flexionextension task. The effect of the biofeedback was almost immediate in some patients, particularly the hemiplegics. They were able to modify their EMG pattern at the first biofeedback session, and over a period of time they were able to maintain this change independent of feedback. In addition, the altered pattern of motor activation was preserved when they were retested one month following the end of the twice weekly program of biofeedback sessions.

It should be emphasized that this was an uncontrolled study. Although the interspersion of training runs with and without visual feedback allowed each subject to serve in a sense as his
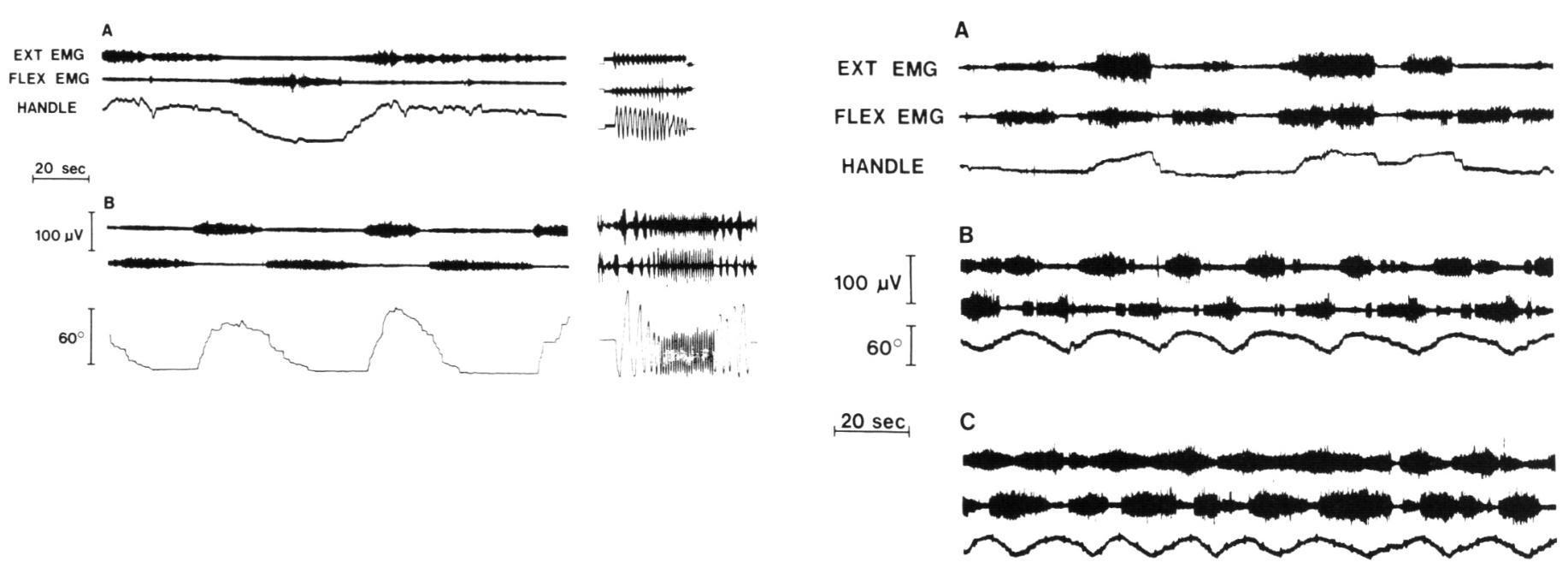

Figure 4 - EMG and associated handle movements in a patient with cerebellar incoordination (case 3 ) before (A) and after (B) five weeks of biofeedback training. The longer segments of recording represent slow tracking movements with visual feedback. The short segments on the right are rapid flexionextension movements performed without feedback.

Figure 5 - Case 4 - spinocerebellar degeneration. A) Recording of EMG and handle position during slow wrist flexionextension movements with visual feedback at the onset of training. Note the prominent coactivation of flexors and extensors with attempts to move the handle. B) Recording with visual feedback after four training sessions. C) Same as B but without visual feedback. 
own control, a control group of subjects who did not receive any biofeedback training was not studied. The small number of patients and the fact that they were not a homogeneous group with respect to the type of motor deficit precludes analysis of the data using conventional statistical methods. The results indicate that these patients were able to modify patterns of EMG activity during biofeedback sessions, but no conclusions can be reached at this stage regarding functional improvement or the long-term pratical advantages of using biofeedback to treat patients with motor disorders. Clearly, what is required is a larger study involving more patients of one diagnostic category and a control group which does not receive feedback.

Several questions must be considered before determining whether these results justify a more extensive trial of EMG biofeed back of the type
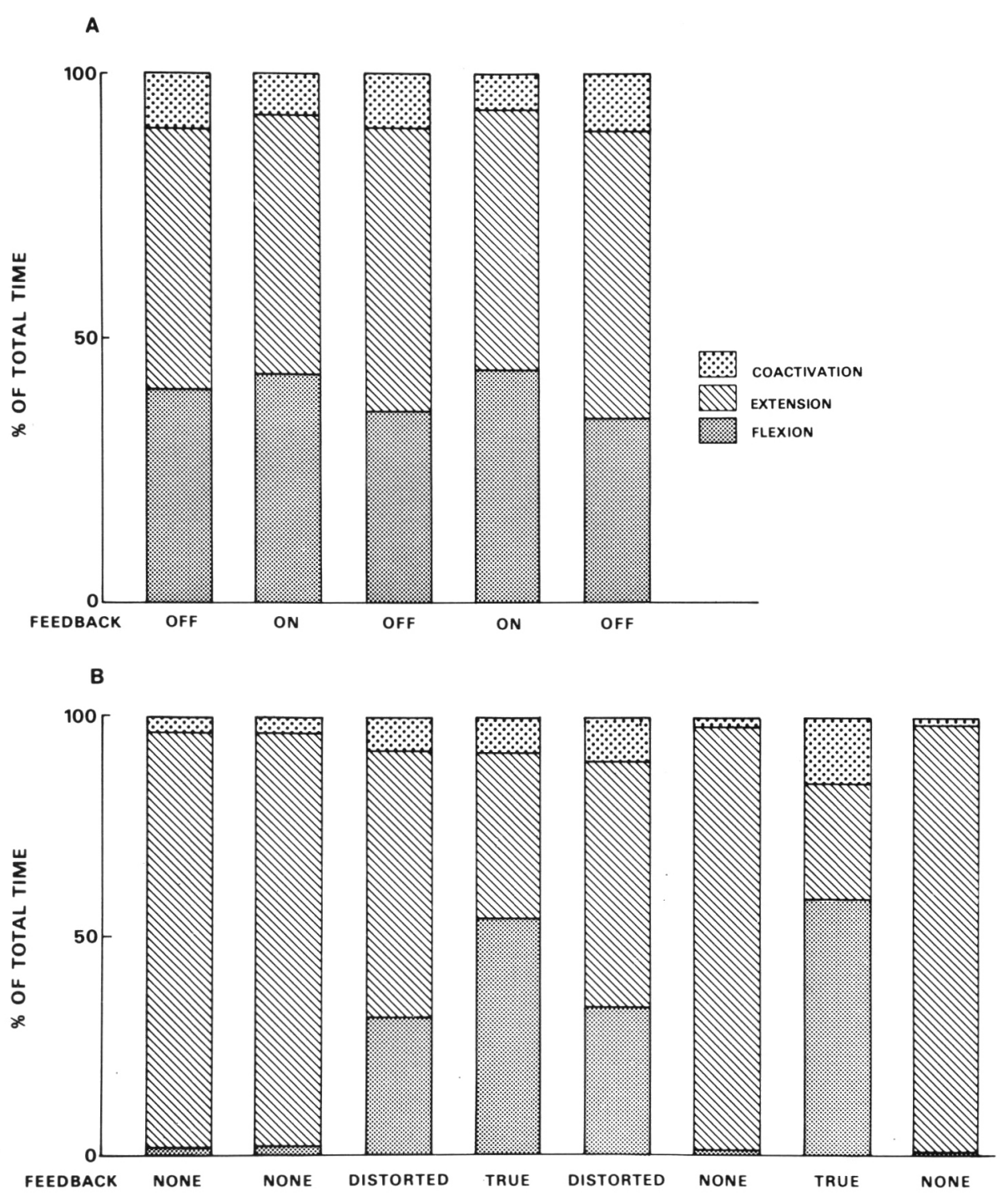

Figure 6-Graphical representation of the percentage of total time allocated to activation of extensors, activation of flexors, and coactivation (see methods). Each bar represents EMG activity obtained during a five minute run of wrist flexion-extension movements with or without visual feedback as indicated at the bottom of the graphs. Results are shown for two patients with cerebellar incoordination: A - case 4, B - case 5 . For case 5 results are also shown for runs using distorted feedback (see text).

we have used. First, was the apparent improvement in EMG activation directly related to the information provided by biofeedback or could it have been due to associated factors? The exposure to rather complex electronic and computing equipment was a novel experience for the patients and might conceivably introduce a placebo effect. The attention focused on the patient and his hemiplegic or dysmetric arm might also lead to an increased level of motivation which could produce the results observed.

Several factors suggest that the changes observed with biofeedback were more than a nonspecific placebo effect. All five patients studied had stable motor deficits which had not changed for at least several months despite prolonged periods of conventional physiotherapy. Also, a clear difference was noted in the EMG pattern during sequential runs with and without feedback. If the changes were due merely to increased attention or motivation they should have been present during the runs without feed back. Furthermore, case 5 was not able to perform nearly so well when provided with distorted feedback, suggesting that the alteration in EMG pattern occurring with feedback was due to the specific information contained in the true feedback signal.

Can EMG biofeedback lead to improved performance on everyday motor functions? Obviously, if this approach is going to be of practical value in rehabilitation of patients with motor deficits it must be shown that the improved patterns of motor activity which develop during feedback can be transferred to everyday tasks. This question was not explored in depth during the present study. However, four of the five patients did show definite improvement of the speed and rhythmicity of rapid voluntary flexion-extension movements of the wrist carried out without feedback. Preliminary work has been undertaken in our laboratory to develop a battery of functional tests to provide a quantitative assessment of upper extremity motor control, and it is intended to incorporate these tests into future studies to compare motor function before and after biofeedback training. 
Assuming that biofeedback can result in some modification of patterns of EMG activation and associated movements, what are the mechanisms responsible for these changes? The simplest explanation is that visual or auditory feedback provides the central nervous system with information concerning the moving extremity which is lacking as a result of the lesion or disease process. Many normal motor functions are dependent on continuous feedback of data concerning the movement which is taking place. The importance of this feedback becomes obvious when one observes the striking abnormalities in motor control which occur in a patient who has lost proprioceptive sensation as a result of lesions in the peripheral nerves, dorsal columns, or somatosensory cortex. Even with no involvement of efferent motor pathways, these patients have considerable difficulty carrying out coordinated movements, particularly if they are deprived of visual input. In this situation feedback of appropriate information via alternative sensory pathways should be able to at least partially substitute for the missing proprioceptive cues.

But how might biofeedback operate in other types of motor deficits in which there are no obvious losses of proprioception or other sensory modalities? In addition to the direct feedback pathway discussed above, there are several other feedback loops which project to cortical motor centres. For example the cerebellum, which receives extensive input from both the cerebral cortex and from peripheral receptors, sends projections back to motor cortex, and defects in this "internal" feedback system are likely responsible for cerebellar dysmetria (Murphy et al, 1975).

If biofeedback is to be effective one must determine which particular properties of movement or muscle contraction are most likely to provide useful information, and how this information can be processed and packaged in a format which can be best utilized by the central nervous system. In the present study we used a visual signal which represented the relative EMG power in agonist and antagonist muscle groups. Whether this even remotely resembles the type of infor- mation transmitted to motor control centers by normal feedback mechanisms is uncertain. Other parameters which could have been selected for feedback include position of the limb, velocity or acceleration of movement, and force of contraction. Further studies are required to determine if some of these parameters might be more appropriate for feedback than EMG activity for certain types of motor disorders.

The possible long term benefits of biofeedback therapy have to be considered in the context of the mechanisms responsible for spontaneous functional recovery following an acute focal cerebral lesion such as an infarct. Early improvement can be attributed to factors such as resolution of cerebral edema and restoration of function in areas which were rendered functionally inactive due to ischemia but which did not undergo necrosis. However, recovery can continue for many months following a stroke, and the mechanisms responsible for these late changes are not well understood. Some of the mechanisms which have been considered include removal of active inhibitory influences, collateral sprouting from surviving axons with formation of new synapses, transfer of functions to undamaged areas of cerebral cortex, the employment of alternative pathways, and the development of new movement strategies (Luria et al, 1969; Geschwind, 1974). Whether any form of biofeedback is capable of influencing these mechanisms remains entirely speculative at the moment, but not outside the realms of possibility.

Two important considerations in evaluating any new form of therapy are acceptability to patients and economic feasibility. In the present study, all patients adapted readily to the procedure and to the concept of interacting with a computer, something which should not be surprising when one considers the recent popularity of electronic games which utilize a television display. The increasing availability of low cost microprocessors and miniaturized electronic circuits should make it feasible to carry out more extensive clinical trials designed to answer some of the questions which have been raised concerning the role of
EMG biofeedback in rehabilitation of motor disorders.

\section{APPENDIX}

The computer program for analysis of EMG activity and generation of the visual feedback display performed the following operations. Flexor and extensor EMG signals were digitized at a rate of 400 samples per second for 100 millisecond epochs. To measure power, the variance of each channel was calculated over the 40 points of the 100 millisecond epoch. For flexor EMG sample $F i(i=1,40)$ and extensor EMG sample Ei $(i=1,40)$, the variances were:

$$
\begin{aligned}
& \operatorname{VAR}(\mathrm{FLEX})=\sum_{i=1}^{40}(\mathrm{~F} i-\overline{\mathrm{F}} i) / 20 \\
& \operatorname{VAR}(\mathrm{EXT})=\sum_{i=1}^{40}(\mathrm{E} i-\overline{\mathrm{E}} i) / 20
\end{aligned}
$$

The EMG vector could have been defined directly in rectangular coordinates using the flexor and extensor EMG variance as the $x$ and $y$ coordinates. However, this would have resulted in a vector moving from $0^{\circ}$ for pure flexion to $90^{\circ}$ for pure extension. To make the display more cognitively useful, the EMG vector was transformed so that $0^{\circ}$ represented pure flexion, while $180^{\circ}$ represented pure extension. This was done by first converting the EMG vector to polar coordinates $(\mathrm{R}, \theta)$ :

$R=\sqrt{V^{-1} R^{2}\left(\text { FLEX) }+V^{2} R^{2}(E X T)\right.}$ $\theta=\mathrm{TAN}^{-1}[\mathrm{VAR}($ FLEX)/VAR(EXT)]

The angle $\theta$ was then multiplied by 2 to give a full display range of $0^{\circ}$ to $180^{\circ}$.

The length $R$ and the angle of $2 \theta$ were then used to define the EMG vector on the display. This process was repeated each 100 milliseconds. Simultaneously the variances for flexor and extensor EMG were stored for later analysis, and the target was stepped across the display once the EMG vector was positioned by the subject within the target boundary.

This work was supported by Medical Research Council of Canada Grant MA 4209. Alan E. Davis was an M.R.C. Post-Doctoral Fellow during the course of this investigation. 


\section{REFERENCES}

ALBANESE, H. and GAARDNER, K. (1977). Biofeed back treatment of tardive dyskinesia: two case reports. Am. J. Psychiat. 134, 1149 1150

BASMAJIAN. J.V., KUKULKA, C. G., NARAYAN, M.G. and TAKEBE, K. (1975). Biofeedback treatment of foot-drop after stroke compared with standard rehabilitation technique: effects on voluntary control and strength. Arch. Phys. Med. Rehabil. 56. 231-236.

BASMAJIAN, J.V., REGENOS, E.M. and BAKER, M.P. (1977). Rehabilitating stroke patients with biofeedback. Geriatrics 32.8588.

BASMAJIAN, J.V. (Ed.) (1979). Biofeedback Principles and Practice for Clinicians. Williams and Wilkins, Baltimore.

BIRD, B.L. and CATALDO. M.F. (1978) Experimental analysis of EMG feedback in treating dystonia. Ann. Neurol. 3, 310-315.
BRUDNY, J., KOREIN, J., LEVIDOW, L., et al. (1974). Sensory Feedback therapy as a modality of treatment in central nervous system disorders of voluntary movement. Neurology 24, 925-932.

BRUDNY, J., KOREIN, J. and GRYNBAUM, B. B. (1976). EMG feedback therapy: review of treatment of 114 patients. Arch. Phys. Med. Rehabil. 57, 55-61.

GESCHWIND, N. (1974). Late changes in the nervous system: an overview. In Plasticity and Recovery of Function in the Central Nervous System. Edited by D. G. Stein, J.J. Rosen and N. Butters. pp. 467-508. Academic Press, New York.

HULTBORN, H., ILLERT, M. and SANTINI, M. (1976). Convergence on interneurons mediating the reciprocal IA inhibition of motoneurons III. Effects from supraspinal pathways. Acta Physiol. Scand. 96, 368-391.

KOREIN, J. and BRUDNY, J. (1976). Integrated EMG feedback in the management of spasmodic torticollis and focal dystonia: a prospective study of 80 patients.
In The Basal Ganglia. Edited by M. D. Yahr. pp. 385-424. Raven Press, New York.

LEE, K. H., HILL, E., JOHNSTON, R, and SMIEHOROWSK1, T. (1976). Myofeedback for muscle retraining in hemiplegic patients. Arch. Phys. Med. Rehabil. 57, 588591.

LURIA, A. R., NAYDIN, V. L., TSVETKOVA, L.S. and VINARSKAYA, E.N. (1969). Restoration of higher cortical function following local brain damage. In Handbook of Clinical Neurology. Edited by P.J. Winken and G. W. Bruyn. Volume 3. North Holland, Amsterdam.

MURPHY, J.T., KWAN, H.C., MACKAY, W.A. and WONG, Y.C. (1975). Physiological basis of cerebellar dysmetria. Canad. J. Neurol. Sci. 3, 279-284.

TERZUOLO, C.A., SOECHTING, J.F. and VIVIANI, P. (1973). Studies on the controls of some simple motor tasks 11. On the cerebellar control of movements in relation to the formulation of intentional commands. Brain Res. 58, 217-222. 